However, ERCP occasionally fails owing to surgically altered anatomy or duodenal stenosis. Endoscopic ultrasound-guided biliary drainage (EUS-BD) has emerged as an alternative biliary drainage method after failed ERCP. We report our practices of EUS-BD, including its technical success rate, clinical success rate, and occurrence of adverse events.

Methods We retrospectively reviewed 19 patients who underwent EUS-BD between February 2017 and July 2018 in Beijing Friendship Hospital.

Results In total, 19 patients underwent EUS-BD (median age 70). The reasons for EUS-BD were altered anatomy after surgery in $7 / 19$, failed ERCP in $7 / 19$, and duodenal stenosis in $5 / 19$. Causes of biliary obstruction included pancreatic cancer (7/19), cholangiocarcinoma(6/19), choledocholithiasis(3/19), postoperative anastomotic stenosis(2/19), and gallbladder cancer(1/19). Biliary drainage was completed by EUS-transmural stenting in $15 / 19$, by EUS-rendezvous technique in $3 / 19$, and by EUS-antegrade stenting in $1 / 19$. Overall technical success was achieved in 18/19, while clinical success was achieved in $16 / 19$. Adverse events included abdominal pain(5/19), cholangitis(4/19), bile leak(1/19), and death(1/19), most of which improved with conservative treatment. Of the three unsuccessful patients, two developed pan-peritonitis and improved after percutaneous transhepatic biliary drainage (PTBD) or surgery, respectively. Another patient died of pancreatic cancer shortly after EUS-BD (table 1).

\begin{tabular}{ll}
$\begin{array}{l}\text { Abstract IDDF2019-ABS-0195 Table } 1 \\
\text { ultrasound-guided biliary drainage(EUS-BD) }\end{array}$ & Outcomes of endoscopic \\
\hline Stenting technique, $\mathrm{n}(\%)$ & $15(78.9)$ \\
\hline Transmural stenting & $3(15.8)$ \\
Rendezvous technique & $1(5.3)$ \\
Antegrade stenting & \\
Punctured site, $\mathrm{n}(\%)$ & $10(52.6)$ \\
Duodenum & $9(47.4)$ \\
Stomach & \\
Deployed stent, $\mathrm{n}(\%)$ & $17(89.5)$ \\
Plastic stent & $2(10.5)$ \\
Metal stent & $94.7 \%(18 / 19)$ \\
Technical success rate & $84.2 \%(16 / 19)$ \\
Clinical success rate & \\
Adverse events, $\mathrm{n}(\%)$ & $5(26.3)$ \\
Abdominal pain & $4(21.1)$ \\
Cholangitis & $1(5.3)$ \\
Bile leak & $1(5.3)$ \\
Death &
\end{tabular}

Conclusions EUS-BD has a high technical and clinical success rate, and a low adverse events rate.

\section{IDDF2019-ABS-0197 RISK FACTORS AND CLINICAL CORRELATES OF NEOPLASTIC TRANSFORMATION IN GASTRIC HYPERPLASTIC POLYPSIN CHINESE PATIENTS}

${ }^{1} \mathrm{Haiyi} H \mathrm{u}^{*},{ }^{1}$ Qian Zhang, ${ }^{1}$ Guangyong Chen, ${ }^{2} \mathrm{D}$ Mark Pritchard, ${ }^{1}$ Shutian Zhang. 'Beijing Friendship Hospital, Capital Medical University, China; ${ }^{2}$ Department of Cellular and Molecular Physiology, Institute of Translational Medicine, University of Liverpool, UK

10.1136/gutjnl-2019-IDDFabstracts. 193
Background Gastric hyperplastic polyps (GHPs) have a potential risk of neoplastic transformation, but the responsible mechanisms have not yet been established.

Methods We conducted a study involving 55 patients (33 female) who had undergone endoscopic or surgical resection of GHPs. We compared 16 patients who had GHPs showing neoplastic transformation with 39 patients who had non-neoplastic GHPs. We analyzed differences in serology, gastroscopic manifestations and pathology between the two groups in order to establish risk factors that may be associated with neoplastic transformation.

Results The mean age of the cohort was $61.73 \pm 9.024$ years. The prevalence of positive serum gastric parietal cell antibody (PCA) was 61.8\%. 30 of the GHPs with neoplastic formation had a "strawberry-like" appearance with erosions of polyps $(P$ $=0.000)$. A history of anemia was a risk factor for GHPs which demonstrated neoplastic transformation (odds ratio [OR], 3.729; 95\% confidence interval [CI], 1.099-12.649; P $=0.035)$. Although the differences were not significant, our data showed higher prevalences of positive serum PCA $(P=$ $0.057)$, hypergastrinemia $(P=0.062)$ and female gender $(P=$ $0.146)$ in the GHP patients who had neoplastic transformation. Multiple polyps in the corpus $(P=0.024)$, iron deficiency anemia $(P=0.036)$, hypergastrinemia $(P=0.000)$ and female gender $(P=0.041)$ occurred more frequently in serum PCA positive patients. Hypergastrinemia occurred more frequently in Helicobacter pylori negative patients and of these 20/22 patients had a positive PCA $(P=0.007)$.

Conclusions GHPs are associated with autoimmune metaplastic atrophic gastritis (AMAG). AMAG is probably one of the risk factors for GHPs to undergo neoplastic transformation. Hypergastrinemia may contribute towards gastric carcinogenesis in these patients.

\section{IDDF2019-ABS-0198 DOUBLE BALLOON ENTEROSCOPY: EXPERIENCE FROM A TERTIARY CARE CENTRE IN SOUTHERN CHINA}

Yi Lu*, Chu-jun Li, Wei-Jie Zhong, Jia-Chen Sun. Department of Gastrointestinal Endoscopy, The Sixth Affiliated Hospital of Sun Yat-sen University, China

\subsection{6/gutjnl-2019-IDDFabstracts. 194}

Background Literature on the experience of double balloon enteroscopy (DBE) is relatively rare, and we conducted this research to share our experience in a tertiary care centre in southern China.

Methods From November 2013 to August 2018, consecutive patients who had a suspicion of small bowel diseases and underwent DBE in our hospital were included in this study. A manual chart review was performed to acquire the following data: age, gender, symptoms, medical histories, laboratory tests, imaging results, endoscopic findings, pathology results, therapeutic interventions, insertion route, length of insertion, procedure time, complications after DBE, the final diagnosis, treatments.

Results After a thorough search, 630 procedures (472 patients) were chosen. The median age of the patients was 31.5 years and $337(71.40 \%)$ were males, while 135 (28.60\%) were females. The main chief complaints of the patients included abdominal pain (167 patients, 35.38\%), diarrhea (57 patients, 12.08\%), gastrointestinal bleeding (121 patients, 25.64\%), weight loss (75 patients, 15.89\%), perianal diseases (33 